\title{
Decay of travelling waves in dissipative Poisson systems
}

By E. van Groesen ${ }^{1}$, F. P. H. van Beckum ${ }^{1}$ and T. P. Valkering ${ }^{2}$, University of Twente, NL7500 AE Enschede, The Netherlands

\section{Introduction}

The specific example to be considered here are 1D wave equations that can be written as a Hamilton (or Poisson) system with Hamiltonian $H$. Then travelling wave solutions can often be found if the system is translationally invariant. This invariance under the (continuous) translation group leads to the existence of an integral, $C$ say, and the profiles of travelling waves are found by looking for critical points of $H$ on level sets of the integral $C$ :

$$
\operatorname{crit}\{H(u) \mid C(u)=\gamma\} .
$$

In this way often a two-parameter family of travelling waves is found:

$$
\{U(\gamma, \varphi) \mid \gamma, \varphi\},
$$

where $\varphi$ is a variable accounting for translation. This $2 \mathrm{D}$ manifold in the state space is called the travelling wave surface (TWS) and is an example of a manifold consisting of relative equilibria of a Poisson system, as shall be shown in the following.

When dissipation is added to the system, these travelling waves are no longer exact solutions. Nevertheless, it is often observed (experimentally and numerically) that there are dissipative motions which at each instant of time look like a travelling wave of the non-dissipative system.

[In fact, the popular quotation of Scott Russel from his "Report on Waves", [Rus1844], which description is usually considered as a first observation of a solitary wave, should actually be interpreted in the sense described above. Indeed, the presence of dissipation was well-observed in this description: "I followed it on horseback, ... Its height gradually diminished, and after a chase of one or two miles I lost it in the windings of the channel."] 
This effect of dissipation is investigated by looking for solutions in a neighbourhood of the travelling wave surface, i.e. for solutions of the form

$$
u(t)=U(\gamma(t), \varphi(t))+\eta(t),
$$

where $\eta$ is "small".

Equations for the evolution of the parameters $\gamma$ and $\varphi$ are derived. These equations provide a two-dimensional approximate description of the system: $u(t)$ is approximated by a dissipative trajectory in the two-dimensional travelling wave surface (2).

Results of numerical simulations are presented that show how well the two-dimensional model approximates the full equations, at least in the standard example of the Korteweg-de Vries equation, supplemented with viscosity (the KdV-Burgers equation), or friction (uniform damping).

With the general method described above, other problems have already been considered. In [Gro88] the self-organisation process of the 2D NavierStokes equations is described as a projection of the dissipative motion on the manifold of relative equilibria of the Euler equations, and describes the results of Foias \& Saut [FS84] in that setting. The evolution of a confined vortex-region along these lines is described in [EG89]. For finite dimensional systems of particle chains, the results in [VDG89] are comparable to those obtained here; see also [BV90] and, for systems with periodic forcing [OV89].

Compared to other work in the literature, the present work combines two different methods that are well-known. First, for completely integrable systems, the use of integrals to construct special solutions can already be found in the first papers on the KdV-equation by $\operatorname{Lax}[\operatorname{Lax} 68,75]$, and for the Toda chain by Ferguson, Flaschka \& McLaughlin [FFM82], as well as in many other papers. In all these papers the special solutions are characterised variationally for the system under consideration, but the effect of dissipation or forcing is not considered there.

Secondly, many methods have been used to perform a perturbation analysis around the special solutions of the conservative part. Many of these methods use other quantities than the defining integrals as perturbation variables. We refer to Ostrovsky [Ost83] and [GO81, 83] as typical examples. Our approach in this paper is closely related to methods used by McLaughlin, see e.g. [MS78] and [KM77]. Furthermore, the use of the Hamiltonian flows of the integrals to reduce the dynamics of Poisson systems is known in general terms (see e.g. [AM78] and [Arn76]), but in the presence of perturbations the equations for the evolution of the integral (the "action") and of the variable describing the contribution of the Hamiltonian flow of that integral (the "angle"-variable), are not straightforward to derive. 
For specific systems the approach used in this paper based on Manifolds of Relative Equilibria, may be related with the theory of Inertial Manifolds (see [Tem88] for a recent review), and with the theory of Center Manifolds. We will return to such relations in future papers.

\section{Factorisation of the Hamiltonian flow}

In this section the translation group related to the second constant of motion is used to "factorise" the Hamiltonian flow.

The equation to be considered for $u=u(x, t)$ is of the following general form:

$$
\partial_{\imath} u=\partial_{x} \delta H(u)-v S(u) .
$$

When $v=0$, the system is conservative and admits exact travelling waves, while if $v \neq 0$ the quantity $S(u)$ will account for dissipative effects.

First consider the conservative case, $v=0$ :

$$
\partial_{t} u=\partial_{x} \delta H(u)=: X_{H}(u) .
$$

Here $H$ is the Hamiltonian, a functional of $u, \delta H$ denotes the variational derivative of $H$ with respect to $u$ (i.e. the Euler-Lagrange expression for $H$ ), and $X_{H}$ is the Hamiltonian vectorfield of the functional $H$.

This equation is a Poisson system: the Poisson bracket for functionals $F$ and $G$ is defined by

$$
\{F, G\}(u)=\left\langle\delta F(u), \partial_{x} \delta G(u)\right\rangle
$$

if the set of functions is appropriate. For definiteness, equation (5) will be considered on the linear space

$$
M:=\left\{u(x) \mid u \text { is } 2 \pi \text {-periodic, } \int u d x=0\right\} .
$$

(On $M$, the spatial derivative $\partial_{x}$ is invertible and the system is in fact a Hamiltonian system with a constant symplectic two-form $\omega$ given by $\left.\omega\left(u_{1}, u_{2}\right)=\left\langle u_{1}, \partial_{x}^{-1} u_{2}\right\rangle.\right)$

Translations of functions will be denoted by $T_{\varphi}$ :

$$
T_{\varphi} u(x)=u(x+\varphi) \quad\left(T_{\varphi}: M \rightarrow M\right) .
$$

The Hamiltonian $H(u)$, a functional on $M$, is assumed to be translationally invariant:

$$
H\left(T_{\varphi} u\right)=H(u) \text { for all } \varphi,
$$

which will be the case if the density does not depend explicitly on $x$. 
Differentiating the identity (7) with respect to $\varphi$ there results

$$
\left\langle\delta H(u), \partial_{x} u\right\rangle=0 .
$$

Introducing the functional $C$ by

$$
C(u)=\int \frac{1}{2} u^{2} d x
$$

this can be written like

$$
\{C, H\}=0,
$$

which shows that $C$ is an integral of the system (5) in involution with $H$. In the following it is assumed that $H$ is not a multiple of $C$, i.e. that $C$ and $H$ are linearly independent.

Since $C$ and $H$ Poisson-commute, the corresponding Hamiltonian flows commute (see e.g. [Arn76], or [AM78]).

It is to be noted that the Hamiltonian $C$-flow is nothing but the translation group, described by $T_{\varphi}$, i.e. $\partial_{\varphi} T_{\varphi} u=\partial_{x} \delta C\left(T_{\varphi} u\right)=X_{C}\left(T_{\varphi} u\right)$.

Using the translation group $T_{\varphi}$ we can formally factorise the Hamiltonian flow. We describe this factorisation procedure in some detail. The intuitive idea is to write the complete evolution $u(t)$ as a (time-dependent) translation $T_{\varphi(t)}$ applied to a function $v(t)$ that describes the evolution modulo such translations:

$$
u(t)=T_{\varphi(t)} v(t)
$$

(see Figure 1.a).

Without specifying the function $\varphi$ at the moment, substituting the expression (10) for $u(t)$ in (5) leads to the following equation for $v(t)$ :

$$
\partial_{t} v=\partial_{x}\left[\delta H(v)-\varphi^{\prime} \delta C(v)\right]=X_{H}(v)-\varphi^{\prime} X_{C}(v) .
$$

(For notational convenience, $\varphi^{\prime}$ denotes the time derivative of $\varphi$ ). To arrive at this result one uses the commutativity property

$$
T_{-\varphi} \partial_{x} T_{\varphi}=\partial_{x}
$$

and the equivariance of $\delta H$ and $\delta C$ for the Hamiltonian $C$-flow:

$$
\delta H\left(T_{\varphi}(v)\right)=T_{\varphi} \delta H(v) \text { and } \delta C\left(T_{\varphi}(v)\right)=T_{\varphi} \delta C(v) .
$$

Since $C$ is an integral, it holds that for each $\varphi$ the vectorfield $X_{H}-\varphi^{\prime} X_{C}$ belongs to the tangent space at the levelset of $C$ through $v$. This tangent space is denoted by $T M_{C}(v)$ and has codimension 1 in the tangent space $T M(v)$ at $v$, and is given by

$$
T M_{C}(v)=\{\xi \mid\langle\xi, \delta C(v)\rangle=0\} .
$$




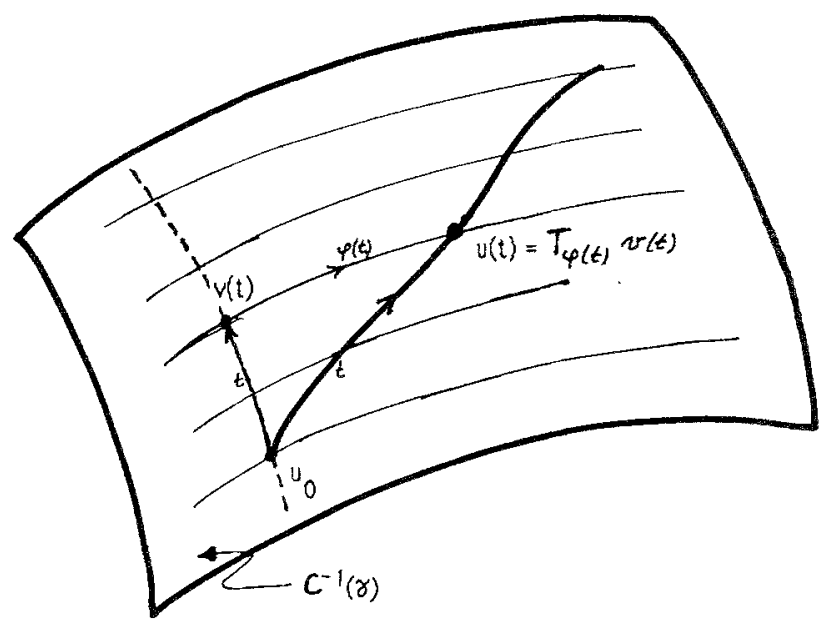

Figure 1.A

Reduction of the dynamics for $u(t)$ on $C^{-1}(\gamma)$ to the dynamcis (18) for $v(t)$ on a Poisson submanifold (codimension 2) by projecting back along the Hamiltonian $C$-flow according to (10).

In the following $T^{*} M(v)$ will denote the cotangent space at $v$. $(T M(v)$ can be identified with $M$ itself at each point $v$, and $T^{*} M(v)$ with the dual space $M^{*}$, but it is helpful in the following to write the point under consideration explicitly.)

To give meaning to the idea that $v$ describes the evolution modulo translations, we choose $\varphi^{\prime}$ in such a way that the right-hand-side of (11) has no component along the Hamiltonian $C$-flow. To specify this, let $n^{*}(v)$ be an element from the dual space $T^{*} M(v)$ such that:

$$
\left\langle\partial_{x} \delta C(v), n^{*}(v)\right\rangle=-1
$$

(For $v \neq 0, \partial_{x} \delta C(v) \neq 0$ and $n^{*}(v)$ exists. See the next remark for a special choice of $n^{*}(v)$, but any $n^{*}(v)$ depending smoothly on $v$ will do.)

Then $\varphi^{\prime}$ is chosen such that

$$
\left\langle\partial_{x}\left[\delta H(v)-\varphi^{\prime} \delta C(v)\right], n^{*}(v)\right\rangle=0,
$$

i.e.

$$
\varphi^{\prime}=-\left\langle\partial_{x} \delta H(v), n^{*}(v)\right\rangle .
$$

With this choice, $\partial_{\imath} v$ satisfies

$$
\left\langle\partial_{t} v, n^{*}(v)\right\rangle=0 \text {. }
$$

This can be formulated in another significant way. From (13) it follows that

$$
\langle\delta C(v), n(v)\rangle=1, \text { with } n(v)=\partial_{x} n^{*}(v),
$$

and so $n(v)$ can be viewed as a normal vector to the tangentspace $T M_{C}(v)$. 
In the same way, $\delta C(v)$ is a normal to the cotangentspace $T^{*} M_{C}(v)$ :

$$
T^{*} M_{C}(v)=\left\{\xi^{*} \mid\left\langle\xi^{*}, n(v)\right\rangle=0\right\} \text {. }
$$

The variational derivative $\delta H(v)$ in $T^{*} M(v)$ can be uniquely decomposed as

$$
\delta H(v)=h^{*}+\mu \delta C(v) \quad \text { with } \quad h^{*} \in T^{*} M_{C}(v)
$$

and $\mu$ the number

$$
\mu=\langle\delta H(v), n(v)\rangle .
$$

Hence $h^{*}=\delta H(v)-\langle\delta H(v), n(v)\rangle \delta C(v)$, and $h^{*}$ can be interpreted as the variational derivative of the restriction $\hat{H}$ of $H$ to the levelset of $C$, and denoted accordingly by $\delta \hat{H}(v)$ :

$$
\delta \hat{H}(v)=\delta H(v)-\langle\delta H(v), n(v)\rangle \delta C(v) .
$$

With this notation, the evolution equation (11) for $v$ can be written like

$$
\partial_{t} v=\partial_{x} \delta \hat{H}(v)
$$

and the equation for $\varphi$ by

$$
\varphi^{\prime}=\langle\delta H(v), n(v)\rangle,
$$

In order to illuminate the reduction procedure described above, introduce the vectors

$$
e_{1}=n(v) \quad \text { and } \quad e_{2}=X_{C}(v)=\partial_{x} \delta C(v) .
$$

As stated before, viewing $e_{1}$ and $e_{2}$ as elements from the tangent space $T M(v), e_{1}$ is normal to the levelset $C^{-1}(\gamma):\left\langle\delta C(v), e_{1}\right\rangle=1\left(e_{1}\right.$ is in the

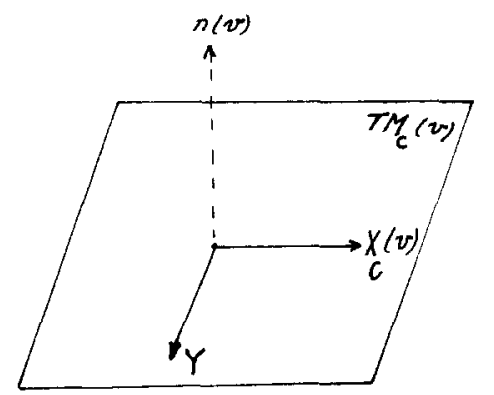

$T M(v)$

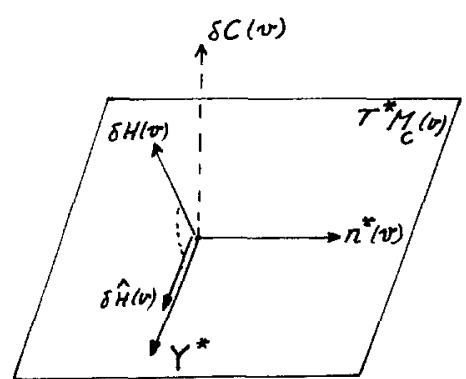

$T^{*} M(v)$

Figure 1.B

The tangent space $T M(v)$ and the cotangent space $T^{*} M(v)$ with the distinguished base vectors $e_{1}=n(v)$, $e_{2}=X_{C}(v), e_{1}^{*}=\delta C(v)$ and $e_{2}^{*}=-n^{*}(v)$, according to (20) and (21). The mapping $\partial_{x}$ satisfies (25) and maps these base vectors into each other. 
gradient direction of $C$ ), while $e_{2}$ is the direction vector tangent to $C^{-1}(\gamma)$ in the direction of the Hamiltonian $C$-flow in $C^{-1}(\gamma)$ (see Figure 1.b).

Dual vectors $e_{1}^{*}$ and $e_{2}^{*}$ that are related to $e_{1}$ and $e_{2}$ by the symplectic operator $\partial_{x}^{-1}$ like

$$
e_{1}^{*}=\partial_{x}^{-1} e_{2} \text { and } e_{2}^{*}=\partial_{x}^{-1} e_{1}
$$

are given by

$$
e_{1}^{*}=\delta C(v) \text { and } e_{2}^{*}=-n^{*}(v),
$$

and satisfy

$$
\left\langle e_{k}^{*}, e_{j}\right\rangle=\delta_{k l}, \quad \text { (duality) }
$$

and so in particular

$$
\left\langle e_{1}^{*}, \partial_{x} e_{2}^{*}\right\rangle=1 \text {. }
$$

The tangent space can be completed with a codimension two space $Y$ orthogonal to $e_{1}^{*}$ and $e_{2}^{*}$ and analogously for the cotangent space:

$$
T M(v)=\left[e_{1}, e_{2}\right]+Y \text {, and } T M^{*}(v)=\left[e_{1}^{*}, e_{2}^{*}\right]+Y^{*},
$$

with

$$
\left\langle e_{k}^{*}, Y\right\rangle=0, \quad\left\langle Y^{*}, e_{j}\right\rangle=0 .
$$

The decomposition of the tangent and cotangent space described here is 'natural' with respect to the action of the mapping $\partial_{x}$. Indeed, it is readily verified that

$$
\partial_{x}: T M^{*}(v) \rightarrow T M(v) \quad \text { with } \quad \begin{aligned}
\partial_{x}: & e_{1}^{*} \rightarrow e_{2}, \\
& Y_{2}^{*} \rightarrow e_{1},
\end{aligned}
$$

With this notation, equation (19) for $\varphi$ is seen to be the projection of the original equation (11) along $e_{2}$, while the remaining equation (18) is the projection of the equation in $Y$. The vanishing of the projection along $e_{1}$ gives

$$
\left\langle\partial_{t} v, \delta C(v)\right\rangle=0,
$$

which expresses the constancy of $C$ along an evolution:

$$
\partial_{t} C(v)=0 \text {. }
$$

This is a clear analytical constraint showing that $v$ is from a set of codimension 1. However there is another restriction to $v$ which shows that $v$ is actually from a codimension 2 set. To see that, note that the choice of $\varphi^{\prime}$ implies that $\partial_{t} v$ satisfies

$$
\left\langle\partial_{t} v, n^{*}(v)\right\rangle=0 \text {. }
$$


Unlike (26), however, this constraint on the derivative of $v$ is usually not easily expressible as an analytical constraint for $v$ (akin to non-holonomic constraints in Classical Mechanics).

Remark. In special cases, including the problem under consideration, it is possible to choose $n^{*}(v)$ in such a way that (27) can be written as the time derivative of a specific functional. This is the case if there exists a functional $Z$ that is canonically conjugate to $C$, by which we mean that

$$
\{C, Z\}=1 \text {. }
$$

Such a functional $Z$ measures the value of $\varphi$ for translations along the $C$-flow:

$$
Z\left(T_{\varphi} v\right)=Z(v)-\varphi,
$$

and can be defined by this property. Then, taking for $n^{*}$ and $n$

$$
n^{*}(v)=\delta Z(v) \text { and } n(v)=\partial_{x} \delta Z(v) \text {, }
$$

the required properties (13) and (16) are satisfied. With this choice, the equation for $\varphi$ reads

$$
\varphi^{\prime}=\{H, Z\},
$$

and the property (27) for $\partial_{t} v$ :

$$
\left\langle\partial_{t} v, n^{*}(v)\right\rangle=-\left\langle\partial_{s} v, \delta Z(v)\right\rangle=0,
$$

can be written like

$$
\partial_{t} Z(v)=0 .
$$

This shows that $v$ in (18) is also constrained to a levelset of $Z$. Since $C$ and $Z$ are linearly independent (since they do not Poisson commute) this makes the reduction of $v$ to a set of codimension 2 explicit.

Clearly, in this case, the integral $C$ and an integral like $Z$ can be interpreted as a pair of "action-angle" variables, with $C$ the action and $Z$ the angle.

In the case under consideration, a functional $Z$ canonically conjugate to $C$ can be written down formally. It is in fact the centre-of-gravity of the density of $C$ :

$$
\int(x-Z(v)) v^{2} d x=0 .
$$

With an integration over the whole real line, this would be the correct expression, uniquely defined, for waves decaying sufficiently fast at infinity. For periodic waves under consideration, integrating over a period usually produces undesired contributions at the boundary. These boundary effects 
vanish if as interval of integration is taken a $2 \pi$ interval for which the endpoints are zero's of $u$. (Each function from $M$ has zero mean, and has therefore at least one zero in each period.) Specifically, if $\alpha(v)$ denotes a zero of $v$ then $Z(v)$ is defined by

$$
\int_{\alpha}^{\alpha+2 \pi}(x-Z(v)) v^{2} d x=0 .
$$

(If $v$ has more zero's, the actual value of $Z(v)$ depends on the choice of the specific choice of the zero. If then $v$ evolves in a continuous way, the evolution of $Z$ should be determined by integrating over the interval translated with the motion of the zero's that have been chosen.)

Indeed, a direct calculation shows that this functional has the desired property: if $\alpha(v)$ is a zero of $v$, then $\alpha(v)-\varphi$ is a zero of $T_{\varphi} v$, and so

$$
\begin{aligned}
\int_{\alpha-\varphi}^{\alpha-\varphi+2 \pi}\left(x-Z\left(T_{\varphi} v\right)\right)\left(T_{\varphi} v\right)^{2} d x & =\int_{\alpha-\varphi}^{\alpha-\varphi+2 \pi}\left(x-Z\left(T_{\varphi} v\right)\right) v^{2}(x+\varphi) d x \\
& =\int_{\alpha}^{\alpha+2 \pi}\left(y-\left[Z\left(T_{\varphi} v\right)+\varphi\right]\right) v^{2}(y) d y
\end{aligned}
$$

from which it follows that $Z\left(T_{\varphi} v\right)+\varphi=Z(v)$.

So, in this case, the evolution of $\varphi$ describes the evolution of the centre-of-gravity and $\varphi^{\prime}$ is the centro-velocity (see also GM89a,b).

The dynamics (18) for $v$ is called the reduced dynamics (Figure 1.a). An element $U$ is called a relative equilibrium for (5) if it is an equilibrium of the reduced dynamics (18), i.e. if $\delta \hat{H}(U)=0$.

In the next section it is shown that these relative equilibria correspond to the travelling waves of the system (5).

\section{The travelling wave surface as a manifold of relative equilibria}

A 'permanent' wave can by definition be written in the form (10) with a function $v$ that is independent of time. Consequently, an equilibrium solution $v=U$ of (18) leads to a solution of the original equation (5) which is a travelling wave solution that propagates with constant phase velocity $\mu(U)$ and has $U$ as its 'profile': $T_{\mu(U) t} U$. Conversely, any travelling wave is necessarily of this form. Since equilibrium solutions of (18) are known as relative equilibria of the original Poisson system, this shows that travelling waves are precisely the relative equilibria.

For $U$ to be a time independent solution of (18), it should hold that $\delta \hat{H}(U)=\alpha$, for some constant $\alpha$. Finding functions $U$ that satisfy this equation, can also be viewed in the following way. 
Consider for $\gamma>0$ the constrained critical point problem:

$$
\operatorname{crit}\{H(u) \mid u \in M, C(u)=\gamma\} \equiv \operatorname{crit}\left\{H \mid C^{-1}(\gamma)\right\} .
$$

It is assumed that this critical point problem does have solutions. This requires some conditions on the functional $H$. General conditions can be formulated for $H$ that assure that $H$ is bounded from below or above and attains a global extremum (For instance, to guarantee the existence of a minimiser, it suffices that $H$ is weakly lower-semi-continuous and coercive on $C^{-1}(\gamma)$ with respect to a topology for which $C^{-1}(\gamma)$ is weakly compact. See e.g. [Ber77].)

Assuming the existence, the following results show that the solutions of this variational problem are the travelling wave profiles of the system and in fact that there is a whole family (parameterized by $\gamma$ ) of such solutions. Moreover, the translation invariance of the system makes it possible to recognize in a natural way a second parameter $\varphi$ in the solutions of (34), which is nothing but a phase shift.

\section{Proposition.}

1]. If $U$ is a critical point of (34) then $U$ satisfies for some multiplier $\lambda$ (and $\alpha)$ the equation:

$$
\delta H(U)=\lambda \delta C(U)+\alpha=\lambda U+\alpha .
$$

2]. For each $\varphi, T_{\varphi} U$ is also a critical point, with the same multipliers $\lambda$ and $\alpha$.

Proof. Equation (35) for $U$ is a direct consequence of the multiplier rule: $\lambda$ is the multiplier from the constraint $C(u)=\gamma$, and $\alpha$ that from the mean zero constraint. Since both $H$ and $C$ are invariant for translations, part 2]. follows.

A consequence of this proposition is that for fixed $\gamma$ there is a oneparameter family $\varphi \rightarrow T_{\varphi} U$. Assuming that the existence is guaranteed of a solution $U(\gamma)$ of (34) for all $\gamma$ in some interval, a two-parameter family of relative equilibria is found:

$$
\left\{U(\gamma, \varphi)=T_{\varphi} U(\gamma) \mid \varphi, \gamma\right\}
$$

As a two-dimensional subset of the state space $M$ this manifold of relative equilibria consists of travelling wave profiles and will therefore be called the travelling wave surface. A specific travelling wave of the Poisson system (5) takes place for a fixed value of $\gamma$ and is a uniform translation with phase velocity $\lambda$ along the (closed) one-parameter curve $\left\{U(\gamma, \varphi)=T_{\varphi} U(\gamma) \mid \varphi\right\}$.

In order to find in the following approximate equations for trajectories near the travelling wave surface in an efficient way, we need some more information about this variational formulation. This is summarised in the 
next Lemma, where for definiteness the critical point problem (34) is assumed to be a minimisation problem.

Lemma. Assume that for each value of $\gamma$ in some interval the constrained minimisation problem

$$
\min \left\{H \mid C^{-1}(\gamma)\right\}
$$

has a solution $U(\gamma)$.

i]. The value-function $h(\gamma)$ of the minimisation problem is well defined by

$$
h(\gamma)=H(U(\gamma)) \text {, with } U(\gamma) \text { (any) solution of } \min \left\{H \mid C^{-1}(\gamma)\right\} \text {. }
$$

Assuming that this function is differentiable, the multiplier $\lambda$ in (35), which in general depends on $\gamma: \lambda(\gamma)$, equals the derivative of $h$ at $\gamma$ :

$$
\lambda(\gamma)=\partial_{\gamma} h(\gamma) \text {. }
$$

ii]. Define $Q=Q(U)$ to be the linear map:

$$
Q(U)=\delta^{2} H(U)-\lambda \delta^{2} C(U),
$$

(note that $\langle Q(U) \xi, \xi\rangle$ is the second variation of $\hat{H}$ in a direction $\xi$ of the tangent space $\left.T M_{C}(U)\right)$. Then it holds that:

$$
Q(U) U_{x}=0,
$$

and

$$
Q(U) U_{\gamma}=\varrho U, \text { where } \varrho=\partial_{\gamma} \lambda(\gamma)=\partial_{\gamma}^{2} h(\gamma) .
$$

Moreover, on the tangent space to the levelset, the second variation is sign definite:

$\langle Q(U) \xi, \xi\rangle \geq 0$ for all $\xi \in T M_{C}(U)$, i.e. for $\xi$ satisfying

$$
\langle\xi, \delta C(U)\rangle=0 \text {. }
$$

Proof. To prove (37), assume for simplicity that there exists a family of solutions $U(\gamma)$ that depends in a differentiable way on $\gamma$. Then

$$
\partial_{\gamma} h(\gamma)=\partial_{\gamma} H(U(\gamma))=\left\langle\delta H(U(\gamma)), U_{\gamma}\right\rangle
$$

where $U_{\gamma}=\partial_{\gamma} U(\gamma)$. Inserting the equation (35) satisfied by a critical point, and using the fact that

$$
\partial_{\gamma} C(U(\gamma))=\left\langle\delta C(U(\gamma)), U_{\gamma}\right\rangle=1, \quad \text { and } \quad \partial_{\gamma} \int U(\gamma)=\left\langle U_{\gamma}, 1\right\rangle=0
$$

the result follows:

$$
\partial_{\gamma} h(\gamma)=\left\langle\delta H(U(\gamma)), U_{\gamma}\right\rangle=\lambda\left\langle\delta C(U(\gamma)), U_{\gamma}\right\rangle=\lambda .
$$


The results (39) and (40) follow by differentiation of (35) with respect to $x$ and $\gamma$ respectively. The non-negativity result (41) is standard for constrained minimisation problems. This completes the proof of the Lemma.

Remark. Concerning the assumption in the Lemma about the differentiability of $h$, the value function of a constrained minimisation problem is under mild conditions Lipschitz continuous and differentiable in a generalised sense (see [Cla83]). Differentiability is assured when, for instance, $h$ is a convex function (see [Gro80]). Instead of proving such a property directly from the abstract minimisation problem, often a more simple way is to prove (the stronger result of) the existence of a family of minimisers $U(\gamma)$ depending smoothly on $\gamma$ by applying the implicit function theorem to the equation (35).

Example. Probably the best known example of an equation of the form (5) is the Korteweg-de Vries equation. It is obtained for the Hamiltonian

$$
H(u)=\int\left[\frac{1}{2} u_{x}^{2}-u^{3}\right] d x
$$

for which

$$
\delta H(u)=-u_{x x}-3 u^{2},
$$

and the equation reads:

$$
\partial_{\imath} u+u_{x x x}+6 u u_{x}=0 .
$$

In this case the constrained critical point problem (34) is a minimisation problem: for each $\gamma>0$ the constrained minimisation problem

$$
h(\gamma)=\operatorname{Min}\left\{\int\left[\frac{1}{2} u_{x}^{2}-u^{3}\right] d x\left|\int \frac{1}{2} u^{2} d x=\gamma, \int u d x=0,\right| u \text { is } 2 \pi \text {-periodic }\right\}
$$

has solutions. These are the well-known cnoidal-wave profiles with fundamental wavelength $2 \pi$. (Other critical points, being saddle points, are cnoidal waves with non-fundamental period $2 \pi / k$, for some integer $k>1$.) If desired, these waves can be written down explicitly in terms of elliptic functions (see. e.g., [Whi74]). The value function is differentiable, and its graph is given in a socalled 'integral-diagram' in Figure 2. This graph can be interpreted as a projection of the TWS on the two-dimensional space spanned by the integrals $H$ and $C$. This integral-diagram will play a key role in the interpretation of the results for perturbed dynamics later on. 
Figure 2

The value-function $h(\gamma)$ of (44): the $T W$-curve (a ID representation of the $T W S$ ). Each point of this curve corresponds to a cnoidal wave with different values of $\gamma$. From the minimisation characterisation (44), no periodic functions with mean value zero can lie below this curve. The tangent to the curve determines the propagation speed of the cnoidal wave, see (37). The tangent at the origin is indicated by the dotted line, and corresponds to the propagation speed of the solution of the linearised system, i.e. of small amplitude waves. Note that in the non linear regime there is a value of $\gamma$ for which the velocity changes sign.

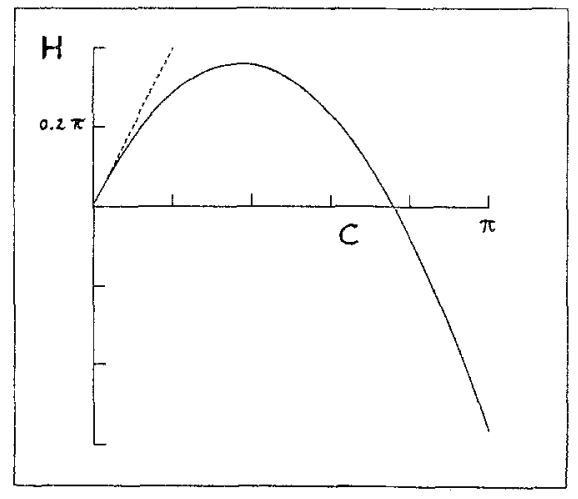

\section{Trajectories of the dissipative equation near the travelling wave surface}

Using the factorisation (10) we derive equations near the travelling wave surface for perturbed equations. The two parameters describing this surface become the basic variables in the transformation of the equations.

Consider the complete equation with $v \neq 0$ :

$$
\partial_{t} u=\partial_{x} \delta H(u)-v S(u) .
$$

For solutions close to the travelling wave surface write

$$
u(t)=T_{\varphi(t)}[U(\gamma(t))+\xi(t)] .
$$

Compared to (3) this means that the perturbation is considered in a frame of reference moving with $\varphi(t)$.

Suppose, in order to guarantee that $\int u d x=0$ for all time for solutions of (4), that the perturbation $S$ satisfies $\int S(u) d x=0$ for all $u \in M$. Inserting (45) into equation (4) leads to the equation

$$
\gamma^{\prime} \cdot U_{\gamma}+\partial_{t} \xi=\partial_{x}\left[\delta H(U+\xi)-\varphi^{\prime} \cdot \delta C(U+\xi)\right]-v S(U+\xi) .
$$

(Again, a prime denotes differentiation with respect to time.) The expression in square brackets can be written like

$$
\begin{aligned}
\delta H(U+\xi)-\varphi^{\prime} \cdot \delta C(U+\xi) & \\
= & \delta H(U)-\lambda \delta C(U)+\left[\delta^{2} H(U)-\lambda \delta^{2} C(U)\right] \xi \\
& \quad-\left(\varphi^{\prime}-\lambda\right) \delta C(U+\xi)+O\left(\xi^{2}\right) \\
= & \alpha+Q(U) \xi-\left(\varphi^{\prime}-\lambda\right) \delta C(U)-\left(\varphi^{\prime}-\lambda\right) \delta^{2} C(U) \xi+O\left(\xi^{2}\right),
\end{aligned}
$$


where $Q$ is the operator defined in (38). The equation becomes

$$
\begin{aligned}
& \gamma^{\prime} \cdot U_{y}+\left(\varphi^{\prime}-\lambda\right) \partial_{x} \delta C(U)+\partial_{t} \xi \\
& \quad=\partial_{x}\left[Q(U) \xi-\left(\varphi^{\prime}-\lambda\right) \delta^{2} C(U) \xi+O\left(\xi^{2}\right)\right]-v \hat{S}(\varphi, U+\xi) .
\end{aligned}
$$

Here, and in the following, $\hat{S}(\varphi, v)$ is defined for notational convenience as

$$
\hat{S}(\varphi, v):=T_{-\varphi(t)} S\left(T_{\varphi(t)} v\right) .
$$

There are several ways to project this equation to the travelling wave surface. This is related to the fact that the function $\xi$ can be chosen from different spaces of codimension 2. A projection based on the symplectic decomposition of the tangent and cotangent space as described in section 2 seems to be the most appropriate in view of the property (25). However, instead of using the base vectors at the point $U(\gamma)+\xi$, we use the base vectors of the nearby point $U(\gamma)$ on the TWS.

Therefore, consider the vectors

$$
e_{1}=U_{\gamma} \quad \text { and } \quad e_{2}=U_{\varphi}=\partial_{x} \delta C(U) \quad\left(=U_{x}\right) .
$$

which are the tangent vectors to the travelling wave surface (see figure 3 ).

Dual vectors $e_{1}^{*}$ and $e_{2}^{*}$ defined as in (21), are given by

$$
e_{1}^{*}=\partial_{x}^{-1} U_{\varphi} \equiv U, \text { and } e_{2}^{*}=\partial_{x}^{-1} U_{y} \text {. }
$$

With spaces $Y$ and $Y^{*}$ as in (23), (24) the decomposition is not only natural with respect to the actions of the mapping $\partial_{x}$ (i.e. satisfies (25)), but also with respect to the operator $Q(U)$ that defines the second variation. Indeed, from the Lemma it follows that

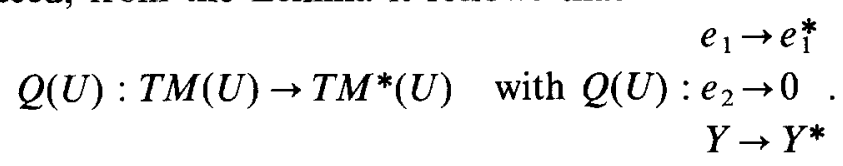

In view of these properties it is natural to choose $\xi \in Y$. Specifically this means that $\xi$ has to satisfy for all time

$$
\left\langle\xi, e_{1}^{*}\right\rangle=\langle\xi, U\rangle=0 \quad \text { and }\left\langle\xi, e_{2}^{*}\right\rangle=\left\langle\xi, \partial_{x}^{-1} U_{\gamma}\right\rangle=0 \text {. }
$$

Figure 3

Direction vectors of the Hamiltonian $C$-flow (translation) and of the gradient $C$-flow as the tangent vectors to the travelling wave surface.

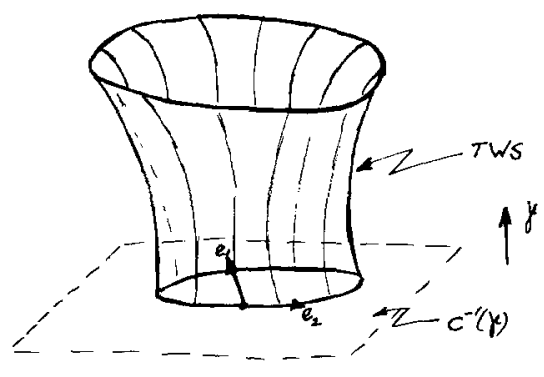


Since that is needed further on, differentiation with respect to time leads to

$$
\left\langle\partial_{t} \xi, U\right\rangle+\gamma^{\prime}\left\langle\xi, U_{\gamma}\right\rangle=0
$$

and

$$
\left\langle\partial_{t} \xi, \partial_{x}^{-1} U_{\gamma}\right\rangle+\gamma^{\prime}\left\langle\xi, \partial_{x}^{-1} U_{\gamma \gamma}\right\rangle=0 .
$$

To investigate the components of equation (47) ( $\equiv E q$ ) along the travelling wave surface, we project this equation along the base vectors $e_{1}$ and $e_{2}$ and along the remaining set $Y$ :

$$
E q=\left\langle E q, e_{1}^{*}\right\rangle e_{1}+\left\langle E q, e_{2}^{*}\right\rangle e_{2}+y, \text { with } y \equiv P_{Y}(E q) \in Y .
$$

Satisfying the equation $E q=0$ requires in the first place the vanishing of the components in the $e_{1}$ and $e_{2}$ direction: $\left\langle E q, e_{1}^{*}\right\rangle=0$ and $\left\langle E q, e_{2}^{*}\right\rangle=0$ leads to (using $(25,50,52)$ :

$$
\begin{aligned}
\gamma^{\prime}+\left\langle\partial_{t} \xi, U\right\rangle & \equiv \gamma^{\prime}\left[1-\left\langle\xi, U_{\gamma}\right\rangle\right] \\
& =-\left(\varphi^{\prime}-\lambda\right)\left\langle\xi_{x}, U\right\rangle-v\langle\hat{S}(\varphi, U+\xi), U\rangle \\
\left\langle\partial_{t} \xi, \partial_{x}^{-1} U_{\gamma}\right\rangle & +\left(\varphi^{\prime}-\lambda\right)=\left(\varphi^{\prime}-\lambda\right)\left\langle\xi, U_{\gamma}\right\rangle-v\left\langle\hat{S}(\varphi, U+\xi), \partial_{x}^{-1} U_{\gamma}\right\rangle
\end{aligned}
$$

The second equation implies that $\varphi^{\prime}-\lambda=O(\xi)+O(v)$, so that we obtain:

$$
\begin{aligned}
& \partial_{t} \gamma=-v\langle\hat{S}(\varphi, U), U\rangle+O\left(\xi^{2}\right)+O(v \xi), \\
& \partial_{t} \varphi=\lambda(\gamma)-v\left\langle\hat{S}(\varphi, U), \partial_{x}^{-1} U_{\gamma}\right\rangle+O\left(\xi^{2}\right)+O(v \xi) .
\end{aligned}
$$

It is to be noted that these equations, apart from the higher order terms, are completely determined from knowledge of the travelling waves.

In an important case, the equation (57) for $\gamma$ does not depend on $\varphi$. This holds if the perturbation $S$ is translationally equivariant (does not depend explicitly on $x): T_{\varphi} S(v)=S\left(T_{\varphi} v\right)$. Then $\hat{S}(\varphi, U)=S(U)$, and, up to higher order terms, equation (57) is a first order differential equation for $\gamma(t)$ only. Solving it, the function $\varphi(t)$ then follows from (58) upon integrating.

With the higher order terms neglected, these evolution equations for $\gamma$ and $\varphi$ describe the projection of the dynamics (4) onto the TWS and can be interpreted as the motion of a damped (nonlinear) oscillator.

Making use of $\partial_{x} Q(U) \xi \in Y$, c.f. $(25,50)$, the remaining equation for $\xi$ reads:

$$
P_{Y}\left(\partial_{t} \xi\right)=\partial_{x} Q(U) \xi-\left(\varphi^{\prime}-\lambda\right) P_{Y}\left(\xi_{x}\right)-P_{Y}(v \hat{S}(\varphi, U+\xi)),
$$

i.e.

$$
P_{Y}\left(\partial_{t} \xi\right)=\partial_{x} Q(U) \xi-\nu P_{Y} \hat{S}(\varphi, U)+O\left(\xi^{2}\right)+O(v \xi) .
$$

We will now present some preliminary observations that may indicate when the perturbation $\xi$ remains "small". 
First note the expected result that a solution with $\xi \equiv 0$ is possible if and only if the dissipation $S$ has only components tangent to the travelling wave surface, i.e. if

$$
P_{Y} \hat{S}(\varphi, U)=0 \text { for all } U=U(\gamma, \varphi) .
$$

This requires $\hat{S}(\varphi, U)$ to be a linear combination of $e_{1}$ and $e_{2}$ :

$$
\hat{S}(\varphi, U)=\alpha U_{\gamma}+\beta U_{x},
$$

where $\alpha$ and $\beta$ may be functionals of $U$. Of course, this needs to hold on the travelling wave surface only. As such it does not provide an easy condition on the kind of dissipation functions $S$ with this property. Nevertheless, as should be expected, it emphasizes the fact that a good approximation of a solution of the complete system by a trajectory consisting of travelling waves is only possible if the dissipation satisfies certain stringent conditions that are related to the specific travelling waves under consideration.

[It is interesting to remark that in an analogous situation for incompressible 2D Navier-Stokes equations, the viscous dissipation does satisfy (61), with $\beta=0$, exactly for functions $U$ that are eigenfunctions of the Laplace operator on the fluid domain. These special solutions (which are relative equilibria in this case) determine for viscous fluids the asymptotic behaviour and describe the 'self-organization process' of such fluids; see [Gro88] and references therein.]

Another way to investigate the $\xi$-equation is to look for a priori estimates that guarantee that $\xi$ remains small for all time when that is the case initially. As it stands, the equation for $\xi$ is a forced linear equation. For boundedness of the solution it must be shown that resonances cannot occur as a result of the primary forcing $P_{Y} \hat{S}(\varphi, U)$. Even more elementary is the requirement that the trivial solution $\xi \equiv 0$ of the unforced problem (i.e. $v=0$ ) should be stable. Of course, this last requirement states that the travelling waves of the conservative system should be (linearly) stable, which is related to the definiteness of the second variation $Q(U)$ on the subspace $Y$.

The next argumentation shows how this last requirement can be exploited to find statements about the small time behaviour of $\xi$.

To that end observe that, since $Q \xi \in Y^{*}$ for $\xi \in Y$, taking the innerproduct of the equation with $Q \xi$ there results:

$$
\left\langle Q \xi, \partial_{t} \xi\right\rangle=\langle Q \xi, v S(U)\rangle+O\left(v \xi^{2}\right)=O(v \xi) .
$$

With

$$
\left\langle Q \xi, \partial_{t} \xi\right\rangle=\partial_{t} \frac{1}{2}\langle Q \xi, \xi\rangle-\left\langle Q_{t} \xi, \xi\right\rangle
$$

and the fact that $Q_{t}=O\left(\gamma^{\prime}\right)=O(v)$ (since the evolution for $\gamma$ is preliminary 
driven by dissipation of order $v$ ), it follows that

$$
\partial_{t} \frac{1}{2}\langle Q \xi, \xi\rangle=v\langle Q \xi, \hat{S}(\varphi, U)\rangle+O\left(v \xi^{2}\right)=O(v \xi)
$$

Consequently, if the constrained critical point problem for $U$ is in fact an extremal problem, in which case $\langle Q \xi, \xi\rangle$ is a norm, the perturbation grows at most with $v$, depending on the component of $Q \hat{S}(\varphi, U)$ in $Y^{*}$.

In that case, starting initially with $\xi=0$, the equations for the trajectory in the travelling wave surface are correct for times satisfying $v t \ll 1$ :

$$
\begin{aligned}
& \partial_{t} \gamma=-v\langle\hat{S}(\varphi, U), U\rangle+O(v t), \\
& \partial_{t} \varphi=\lambda(\gamma)-v\left\langle\hat{S}(\varphi, U), \partial_{x}^{-1} U_{\gamma}\right\rangle+O(v t) .
\end{aligned}
$$

\section{Numerical computations}

For the $\mathrm{KdV}$ equation (43), two types of dissipation were considered: the uniformly damped $K d V$ with friction $S(u)=u$ :

$$
u_{t}+u_{x x x}+6 u u_{x}=-\sigma u,
$$

and the $K d V$-Burgers equation, with viscous dissipation given by $S(u)=-u_{x x}$ :

$$
u_{t}+u_{x x x}+6 u u_{x}=v u_{x x} \text {. }
$$

In both cases, an exact travelling wave of $\mathrm{KdV}$ was taken as initial condition and the (perturbed) dynamical equation was integrated numerically. For various quantities the results were compared with the approximated dynamics on the TWS. Omitting the higher order terms in $(57,58)$, and observing that both perturbations are translationally equivariant, these approximate equations read:

$$
\begin{aligned}
& \partial_{t} \gamma=-v\langle S(U), U\rangle, \\
& \partial_{t} \varphi=\lambda(\gamma)-v\left\langle S(U), \partial_{x}^{-1} U_{\gamma}\right\rangle .
\end{aligned}
$$

For the specific problems at hand, a special symmetry in the equations simplifies the approximations even further: since each waveprofile is symmetric, the expression $S(U) \cdot \partial_{x}^{-1} U_{\gamma}$ is anti-symmetric and vanishes upon integration. Hence the equations on the TWS simply become:

$$
\begin{aligned}
& \partial_{i} \gamma=-v\langle S(U), U\rangle, \\
& \partial_{t} \varphi=\lambda(\gamma) .
\end{aligned}
$$

The qualitative and quantitative comparison with the exact dynamical equations is presented in the following Figures 4-8 for the two cases: $A$. the $\mathrm{KdV}$-equation with friction (65), and $\mathbf{B}$. the $\mathrm{KdV}$-Burgers-equation (66). 
Figure 4.A

Numerical results for the uniformly damped KdVequation (65), with $\sigma=1$ and $\sigma=10$, -the lower and upper solid curve respectively. The dotted curve is the $T W$-curve; for $\sigma=0.1$ the evolution is graphically indistinguishable from this $T W$-curve. For all calculations as initial value was taken the point at the $T W$-curve with $C=3 \pi / 4$.

Figure 4.B

As Fig. 4.A. but now for the KdV-Burgers equation, for $v=1$ and $v=10$. Again, for $v=0.1$ the evolution practically coincides with the $T W$-curve.
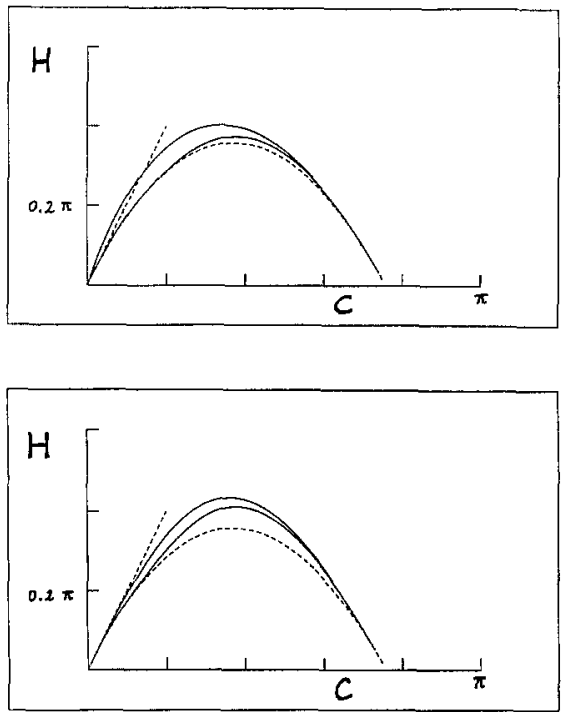
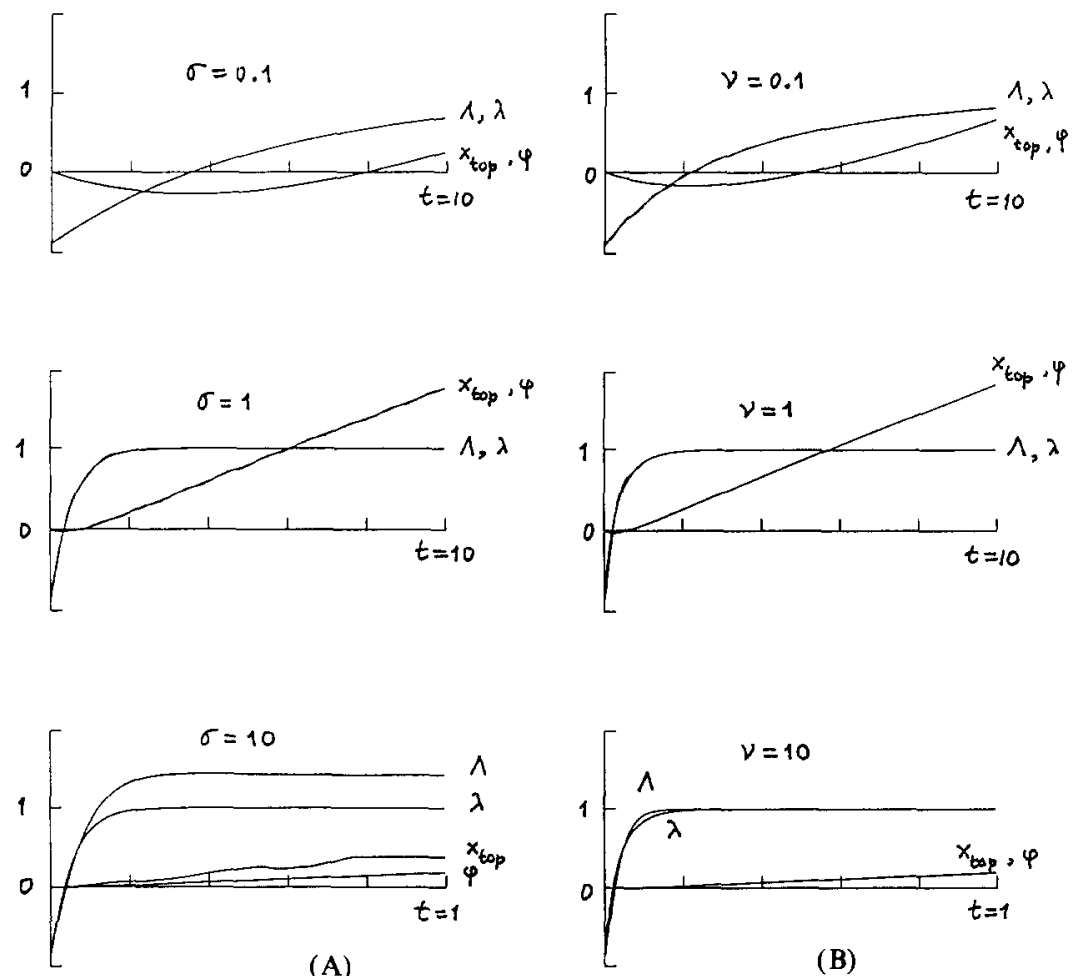

Figure 5

For various values of the dissipation parameters are displayed the value of the velocity of the cnoidal wave $\lambda(\gamma)$ and of the dissipation rate quotient $\lambda(u),(70)$, for the calculated solution, and the value of the position of the top of the calculated solution $x_{\text {top }}$ (found by inspection), and the value of $\varphi_{T w S}$ as it follows from the projected dynamics (68). In figure $A$ for the uniformly damped $\mathrm{KdV}$, and in $\mathrm{B}$ for the KdV-Burgers equation. For $\sigma=0.1$ and $\sigma=1$, and for $v=0.1$ and $v=1$ these curves practically coincide. See Figures 6 and 7 for more detailed information. 
A.

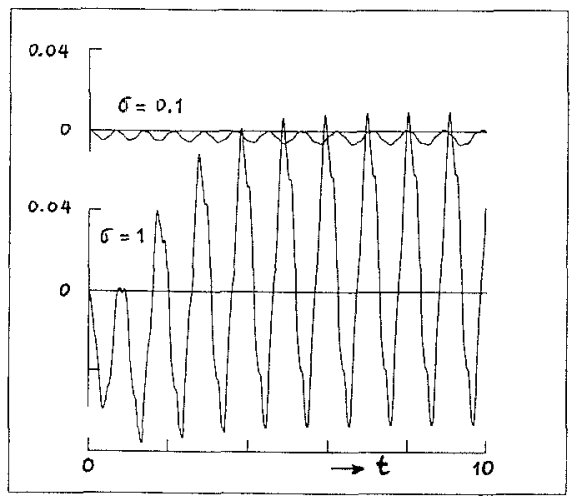

B.

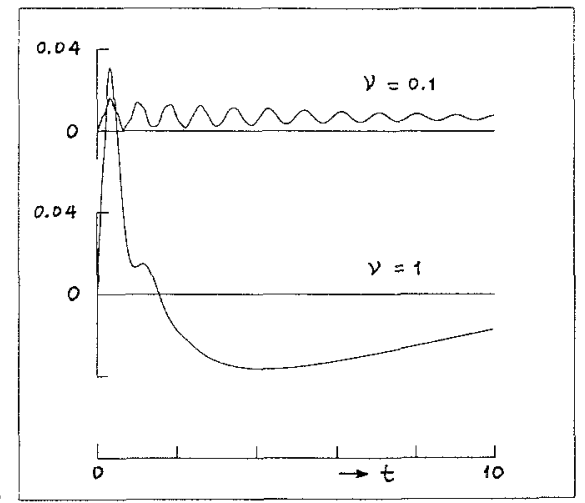

Figure 6

Difference of quantities appearing in Figure 5: $x_{\text {top }}-\varphi_{T W S}$. In figure A for the uniformly damped $\mathrm{KdV}$, and in $\mathbf{B}$ for the $\mathrm{KdV}$-Burgers equation.

In Figure 4 the trajectories in the $H-C$ diagram are displayed for the evolution according to the perturbed equations. Note that for the unperturbed $\mathrm{KdV}$-equation each solution would correspond to a fixed point in this diagram, since $H$ and $C$ are integrals. This is no longer the case in the presence of friction or viscosity, and the values of the functionals $H$ and $C$ will change in the course of time. Figure 4 shows the evolution of these functionals as calculated from the complete equations. By comparison, the projected dynamics (68) leads to a trajectory that is given by the $T W$-curve of $\mathrm{KdV}$ in this diagram. In order to interpret the evolution, two quantities are of special interest: the integral-quotient $Q$ and the dissipation-rate quotient $\boldsymbol{A}$, defined for solutions of the perturbed equations as

$$
Q(u)=H(u) / C(u)
$$

and

$$
\Lambda(u)=\partial_{t} H(u) / \partial_{t} C(u)
$$

which quantities are the direction-vector and the tangent-vector to the trajectory in the integral-diagram respectively. Note that on the $T W$-curve, $\Lambda(u)$ coincides with the value of the multiplier $\lambda$ at that point, and hence denotes the velocity of the wave. Also from the expression (70) it may look as if $\boldsymbol{\Lambda}(u)$ can be interpreted as a kind of wave-velocity. The next figures indicate in more detail the differences between the exact solution and the approximation. In Figures 5, 6 and 7, the horizontal axis is the time. Vertically the value $\boldsymbol{\Lambda}(u)$ of the exact dynamics is compared with the value of the corresponding approximant $\lambda(U)$; also the value of the position of the 'top' of the numerically calculated solution (found by inspection) is compared with the value of $\varphi$ as determined by the approximation (68). In 
A.

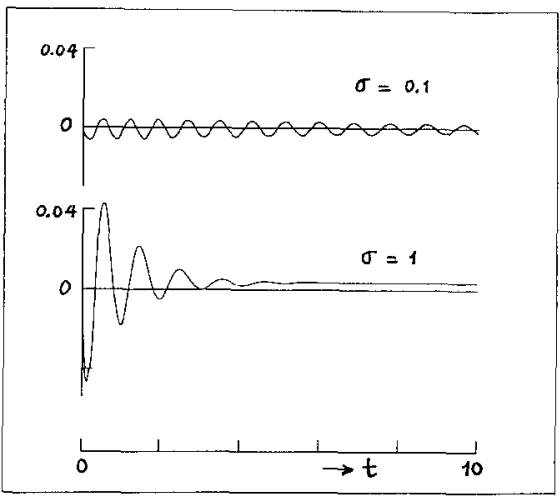

B.

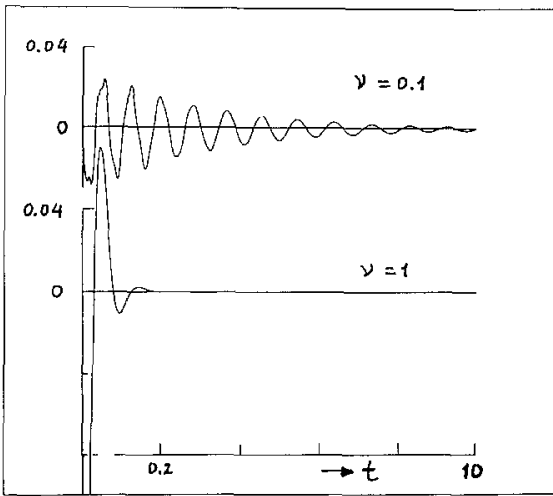

Figure 7

Difference of quantities appearing in Figure 5: $\boldsymbol{A}-\lambda$. In figure A for the uniformly damped $\mathrm{KdV}$, and in $\mathbf{B}$ for the KdV-Burgers equation.

Figure 8 the shape of the 'asymptotic' waveprofile is plotted (normalised by sqrt $C$ and for a typical time $t \gg 1$ ). It is seen, and confirmed by the numerical results presented in Figure 9, that in the viscous case for KdV-B a 'self-organisation' process has taken place: all Fourier modes decay faster than the lowest one, and consequently only the longest wavelength is present for large time. This time-asymptotics can easily be verified analytically, e.g. as in the case of 2D Navier-Stokes equation, see [Gro88]. For the uniformly damped equation (65) this self-organisation does not happen, and in fact the 'asymptotic' waveprofile depends somewhat on the choice of the initial condition. As a quantity to measure this difference the relative

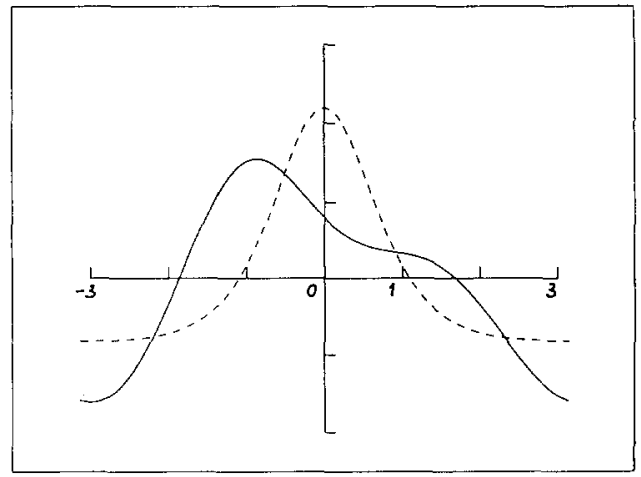

A.

\section{Figure 8}

The 'asymptotic' profile for large values of time (solid), presented as a function of $x$ in a normalized way as $u(x, t) / \sqrt{ } C$, compared with the initial profile (dotted). In figure $\mathrm{A}$ for the uniformly damped $\mathrm{KdV}$, and in $\mathrm{B}$ for the KdV-Burgers equation.

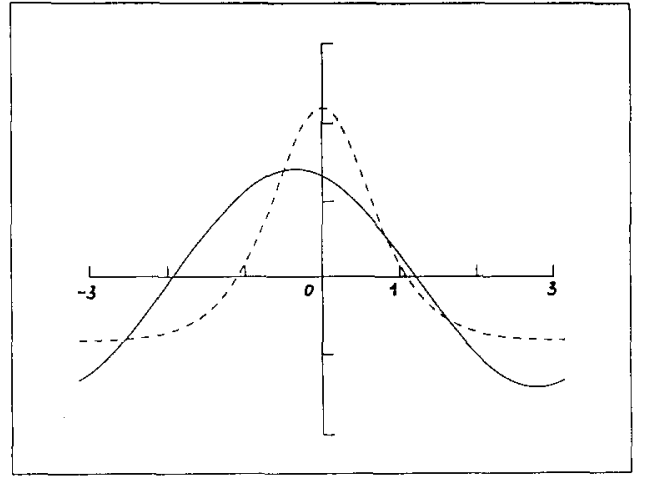

B. 
contribution of the lowest Fourier modes has been displayed: if the solution is represented as

$$
u(x)=\sum_{m=-n}^{n} a_{m} \exp (\operatorname{im} x)
$$

then for $k<n$ this relative contribution reads:

$$
\sum_{m=-k}^{k} a_{m}^{2} / \sum_{m=-n}^{n} a_{m}^{2}
$$

Remark. The numerical integration of the complete equations (65) and (66) were performed using a very accurate discretisation scheme. The scheme consists of a structure-consistent spatial discretisation followed by a fourth-order Runge Kutta time integration. The spatial discretisation is such that it has inherited the Poisson structure when dissipation is ignored. In particular, the integrals $H$ and $C$ have discretisations that are conserved exactly for the space discretised system. Consequently, exact solutions that correspond to the travelling waves, do exist in this discretisation. Computer
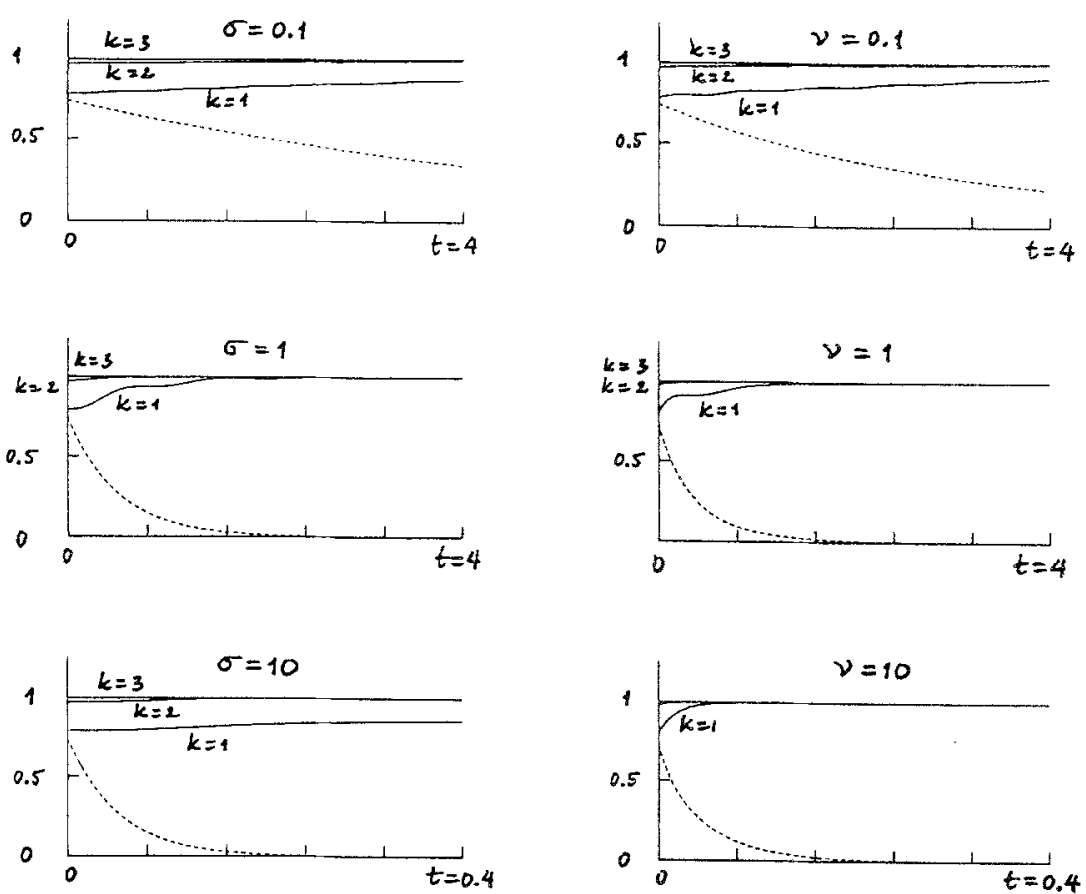

(A)

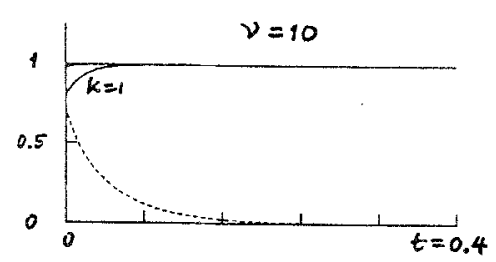

(B)

Figure 9

The relative weight of the lowest order Fourier modes as a function of time for the numerical solution of the complete dynamics. For various values of $k$ the quotient (72) is shown. In figure A for the uniformly damped $\mathrm{KdV}$, and in $\mathrm{B}$ for the $\mathrm{KdV}$-Burgers equation. The dotted curve represents the decay of $C$, indicating the decay of the solution. 
runs showed that in the absence of dissipation, these waves propagated without change of form or change of phase over time intervals that are large compared to the typical times as required for the calculations presented here in the presence of dissipation. See for details of this discretisation procedure [BG87,90].

\section{Acknowledgement}

Remarks of the referee have been very helpful to clarify the presentation.

\section{References}

AM78 R. Abraham and J. E. Marsden, Foundations of Mechanics, 2nd ed. Benjamin/Cummings Publ. Co. 1978.

Arn76 V. Arnold, Methodes Mathématiques de le Méchanique Classique, Editions M1R, Moscou 1976.

Ber77 M. S. Berger, Nonlinearity and Functional Analysis, chapt. 6, Academic Press, New York 1977.

BG87 F. P. H. van Beckum and E. van Groesen, Discretizations conserving energy and other constants of the motion, Proc. ICIAM, pp. 17-35, Paris 1987.

BG90 F. P. H. van Beckum and E. van Groesen, Model consistent discretisations of $1 D$ wave equations (to be published 1990).

BV90 G. Brinkman and T. P. Valkering, Soliton decay in a Toda chain caused by dissipation. ZAMP 41, $61-78$ (1990).

Cla83 F. H. Clarke, Optimization and Nonsmooth Analysis, Wiley, New York 1983.

EG89 A. Eydeland and E. van Groesen, An extended self-organization principle for modelling and calculating the dissipation of $2 D$ confined vortices. Nonlinearity (1989).

FFM82 W. E. Ferguson, H. Flaschka and D. W. McLaughlin, Nonlinear normal modes for the Toda chain. J. Comp. Phys. 45, 157-209 (1982).

FS84 C. Foias and J. C. Saut, Asymptotic behaviour as $t \rightarrow \infty$ of solutions of Navier-Stokes equations and non-linear spectral manifolds. Indiana Univ. Math. J. 33, 459-477 (1984).

GM89a E. van Groesen and F. Mainardi, Balance laws and centro-velocity in dissipative systems. J. Math. Phys., 1990 (in press).

GM89b E. van Groesen and F. Mainardi, Energy propagation in dissipative systems. Wave Motion 11, $459-475$ (1989).

G081 K. A. Gorshov and L. A. Ostrovsky, Interactions of solitons in nonintegrable systems: Direct perturbation method and applications. Physica 3D, 428-438 (1981).

G083 K. A. Gorshov and L. A. Ostrovsky, Nonlinear vector waves in a mechanical model of a molecular chain. Physica $8 D, 223-228$ (1983).

Gro80 E. van Groesen, Dual and inverse formulations of constrained extremum problems. J. Math. Modelling 1, 237-254 (1980).

Gro88 E. van Groesen, Time-asymptotics and the self-organization hypothesis for $2 D$ Navier-Stokes equations. Physica 148A, 312-330 (1988).

KM77 J. P. Keener and D. W. McLaughlin, Solitons under perturbation. Phys. Review 16A, 777-790 (1977).

Lax68 P. D. Lax, Integrals of nonlinear equations of evolution and solitary waves. Comm. Pure Appl. Math. 21, 467-490 (1968).

Lax75 P. D. Lax, Periodic solutions of the KdV equation. Comm. Pure Appl. Math. 28, 141-188 (1975).

MS78 D. W. McLaughlin and A. C. Scott, Perturbation analysis of fluxon dynamics. Phys. Review 18A, $1652-1680$ (1978).

OV89 J. H. J. van Opheusden and T. P. Valkering, Period-doubling density waves in a chain. Nonlinearity 2(1989).

Ost83 L. A. Ostrovsky, Solitons in Active Media. In Nonlinear Deformation Waves. IUTAM Symposium. (Eds U. Nigul and J. Englebrecht), Springer 1983.

Rus1844 J.Scott Russell, Report on Waves. Report on the fourteenth meeting of the Brit. Assn. Adv. Sci., pp. 311-390, John Murray, London 1845.

Tem88 R. Teman, Infinite Dimensional Dynamical Systems in Mechanics and Physics. Springer 1988. 
VDG89 T. P. Valkering, G. Derks and E. van Groesen, Modelling one degree of freedom behaviour of dissipative Hamiltonian systems with forcing. Proc. Singular Behaviour and Nonlinear Dynamics (Eds S. T. Pneumatikos et al.), pp. 457-470. World Scientific Publ. 1989.

Whi74 G. B. Whitham, Linear and Nonlinear Waves. Wiley, New York 1974.

\begin{abstract}
In many finite and infinite dimensional systems low-dimensional behaviour is often observed. That is to say, the dynamics, observed experimentally or numerically, looks as if it can be described (approximately) with only a few essential parameters. Choosing the correct set of such "robust observables" is an essential ingredient of a successful low dimensional description. This paper reports on a specific example of a more general approach that aims at describing certain (low dimensional) phenomena in (high dimensional) damped/driven equations with parameters that are essentially determined from the underlying conservative part of the equation. In particular, a Hamiltonian or a Poisson structure of the conservative part is exploited to find (characterize) families of exact solutions. These solutions are then used as the "base functions" with the aid of which the solutions of the disturbed system are approximated. This approximation is accomplished using the parameters that characterize the family as variables that depend on time. In this paper, this procedure is applied to a class of systems which admit travelling waves when dissipation is ignored.
\end{abstract}

(Received: May 29, 1989; revised: January 12, 1990) 